\title{
Construction and Use of Nansha Islands Digital Science Education Software with Virtual Reality Technology
}

\author{
Kong Yiquan ${ }^{1}$, Jin $\mathrm{YiFu}^{2}$ \\ 1. School of Information Engineering, Lingnan Normal University, Zhanjiang Guangdong 524048 \\ 2. Digital Learning Engineering Technology Development Center of Guangdong College, Zhanjiang Guangdong 524048
}

\begin{abstract}
The project of Nansha islands digital science education software promotes the dissemination of marine culture and awareness of the oceans and provides better marine cultural services to the public. We constructed the system frame of Nansha islands digital science education software (NIDSES). NIDSES designed the function including sea culture popular science with virtual reality technology, ocean geography science somatosensory interaction, and humanistic science with game. We also elaborated the key technology and application scenarios in the NIDSES. Using NIDSES we can know the history, geography and the humanities the Nansha islands and it will be of great importance.
\end{abstract}

Keywords - Virtual reality; Nansha islands; Education software; Polular science

\section{INTRODUCTION}

Virtual reality has been used to describe a variety of methods for turning computers into something other than glorified typewriters. The phrase conjures up images of people plugging into their computers with dis-play goggles and interactive controllers that allow them to experience and move within artificial environments in ways simulator utterly different than those of the real world. Use of virtual reality (VR) has been developing rapidly in the education field. The efficacy and efficiency of VR application in either an immersive or a non-immersive type, has been demonstrated for different client groups during the last few decades [1]. One reason behind why virtual reality is compelling is because it exploits our inherent capacity to experience and use three-dimensional spaces without being limited to the physical world. Applications of VR offer the promise of enhancing human memory and intelligence.

The educational opportunities for VR are almost too obvious to mention. One of the first successful VR experiences is Titans of Space, which lets you explore the solar system first hand. Science, history, arts, and mathematics - VR will help students of all ages because, as they say, field trips are much more effective than textbooks [2].

\section{WHAT IS VIRTUAL REALITY (VR)?}

It is a cutting-edge computer technology which has its origins in visually coupled system and formed the basis of the first flight simulator. VR is a computer generated environment. It was based on computer simulation and real-time visual, auditory manipulate, and interact with computers and extremely complex data. VR could also be viewed as an advanced form of human-computer interface that allowed users to "interact" with and become "immersed" in a computergenerated environment in a naturalistic fashion. Characteristics of virtual reality systems include navigation (exploring, orientating), interaction (opportunities to engage in virtual environment or VE), presence (subjective feeling of being present in a simulated environment) and immersion (objective measure -VR platform, technology-based). VR is now being widely applied in many fields including engineering, architecture, design, medicine, education and training [3]. The potential of virtual environments (VEs) in the field of education has been noted. System architecture of Nansha islands digital science education software

\section{SYSTEM ARCHITECTURE OF NANSHA ISLANDS DIGITAL SCIENCE EDUCATION SOFTWARE}

System architecture of Nansha islands digital science education software (NIDSES) is shown in Figure 1. Use of virtual reality (VR) has been developing rapidly in the education field. The efficacy and efficiency of VR application in either an immersive or a non-immersive type, has been demonstrated for different client groups during the last few decades. We constructed the system frame of Nansha islands digital science education software (NIDSES) and built a virtual reality world and share it with others as a shared social experience. We use one of the numbers of existing social VR applications that provide the infrastructure and allow customization. At the time of writing this book, these include VRChat, VRoom, and others. One of the key factors that support accessibility is the provision of multimodal user interfaces. Multimodality as the possibility of using in parallel more than one interaction modalities for communication between user and system has been implemented in four modes: whereas visual and acoustic modalities are used for system outputs, inputs are achieved through tactile and haptic methods. 
Acoustic input (i.e. voice recognition) was left out for future platform versions [4]. NIDSES uses sensory experience technology, human engineering technology, computer graphics processing technology, network parallel processing technology.

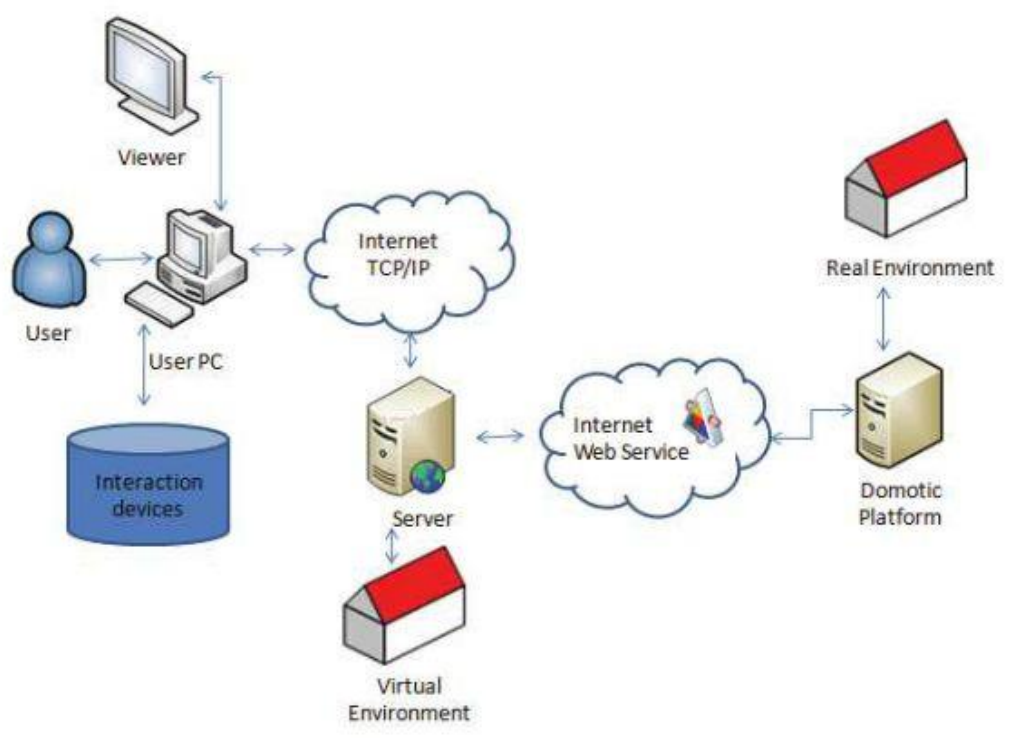

Fig. 1. System architecture of Nansha islands digital science education software

\section{ARTIFICIAL INTELLIGENCE BASED NIDSES VIRTUAL} REALITY PROGRAM

The innovative use of artificial intelligence (AI) techniques such as Case-Based Reasoning has been proposed to develop the VR system allows some flexibility to facilitate individual learning. The main advantage in integrating VR systems with embedded AI software can be the ability of providing instant analysis of the user's behavior. Therefore, immediate feedbacks and assistance can be given to the users in the forms of additional clues and objects in the virtual world. This will allow for individual styles of learning and their relative stages of recovery. A recent development would be an AI based VR system for prospective memory training for education skills. By altering three parameters, namely number of items in the Nansha islands digital science education list $(\mathrm{S})$, number of prospective memory task $(\mathrm{P})$ and level of assistance (A). After gathering the training performance of each trial/session, AI system can plan the level of difficulty in the next session [5]. The system of NIDSES is not only full of entertainment and artistry, but also permeates the knowledge of the ocean and plays a popular role in science and education.

\section{THE FRAMEWORK OF A CONTENT-BASED 3D MODEL RETRIEVAL SYSTEM OF NIDSES}

The framework of a content-based 3D model retrieval system is shown in Figure 2. It is composed of several components: user interface, query processor and 3D geometry database. To build a 3D geometry database, 3D models are first converted into compact and representative features through the feature extraction process. An indexing structure is then built to store the features in an efficient manner [6]. A typical approach for query processing is called query by example. First, the user inputs a sample model. The system extracts the representative features from this model and matches them with each set of the features extracted from the models stored in the 3D geometry database using a feature matching technique. Models in the database with similar features will be returned to the user. To speed up the searching process, the system may employ the nearest neighbor search on the database index to find models that are most relevant to the given query and return retrieval results through the search engine interface. Through the computer background synthesis technology, NIDSES has generated the human body sense, the vision, the auditory manifestation way, causes the operator through the computer external equipment, and enhances to the virtual world the interaction and the experience function. The virtual reality engine provides the data for the sand archipelago sea culture popular science, alternately, the function and the special effect expansion connection, under the realization virtual reality technology the Nansha Islands sea culture popular science visualization simulation function. 


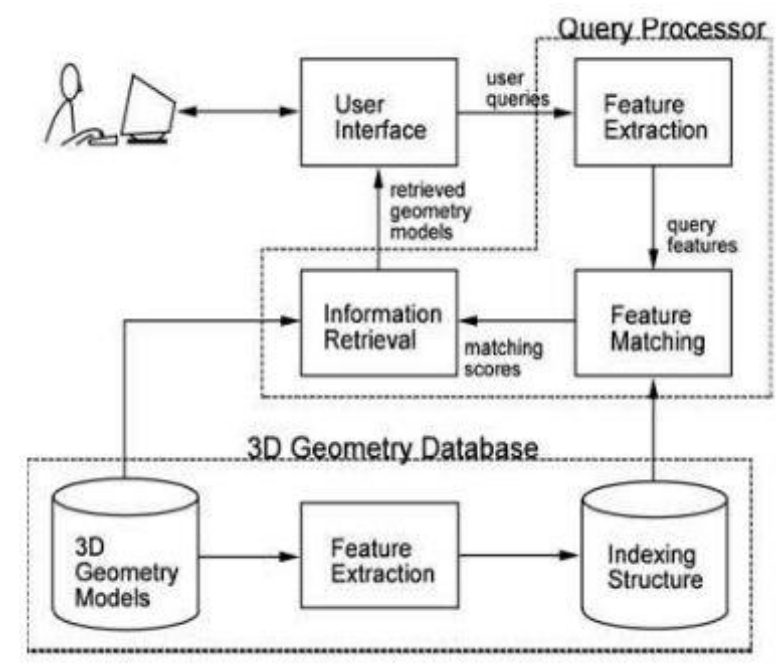

Fig. 2. System architecture of Nansha islands digital science education software

\section{THE PRACTICE OF NANSHA ISLANDS DigITAL SCIENCE EDUCATION SOFTWARE}

NIDSES designed the function including sea culture popular science with virtual reality technology, ocean geography science somatosensory interaction, and humanistic science with game. We also elaborated the key technology and application scenarios in the NIDSES. Using NIDSES we can know the history, geography and the humanities the Nansha islands and it will be of great importance. Under the virtual reality the Nansha Islands sea culture popular science database is the hypothesized visualization data origin, provides the data organization rule and the data support for the three dimensional popular science education environment visualization exaggerations. $3 \mathrm{D}$ visualization and virtual reality technology to its unique immersive, imaginative, interactive atlas of marine cultural visual simulation shows the strong technological advantage.

Application scenarios of Nansha islands digital science education software is shown in Figure 3 and Figure 4. We developed interactive walkthrough, dynamic loading, multi view dynamic browsing, interactive picking and querying in the virtual reality 3D digital model. Through simulation and sea-bottom three-dimensional flight, the distribution and shape of main trenches, Ridge and basin of Nansha Islands are browsed. The dynamic simulation of marine meteorology, wave and tide in Nansha Islands can be realized by tapping the keyboard. We can enjoy a wide range of marine fishes, corals and other colorful marine world in the application scenarios of Nansha islands digital science education software. We are therefore studying ways to introduce the Conceptual Web into other environments. Apart from their usage on the ordinary web, we are investigating the fascinating possibility of introducing conceptual structures in 3D environments. A 3D environment filled with semantics and conceptual structures would present a fundamentally different experience, enabling for the first time a virtual reality full of meaning, and not only packed with dead 3D objects whose meaning is defined by the graphics engine. The system will apply three-dimensional somatosensory interactive digital technology to the Nansha Islands Marine Geography Science popularization field, not only full of entertainment and artistry, but also infiltrate the knowledge of the ocean, play the role of science popularization and entertaining.

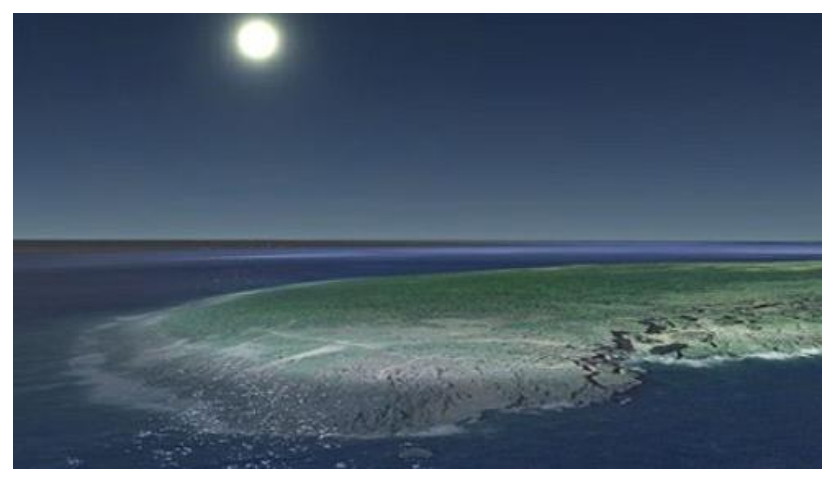

Fig. 3. Application scenarios of NIDSES

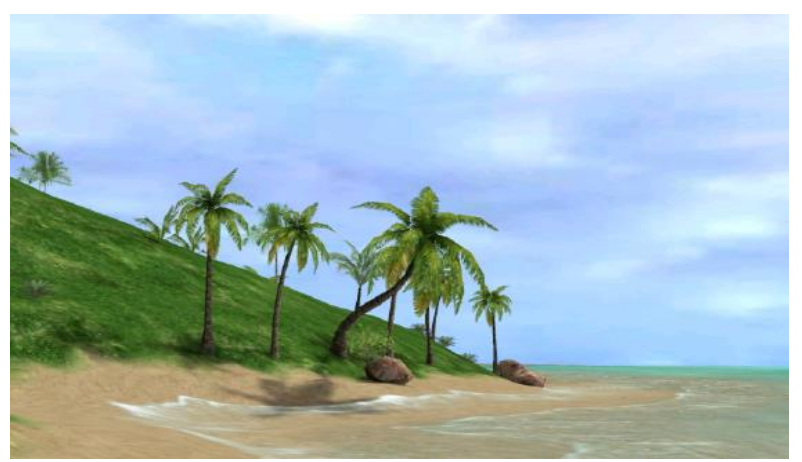

Fig. 4. Application scenarios of NIDSES

\section{SUMMARY}

The Nansha Islands and its neighboring region is Chinese traditional territory. The region has very important strategic value for the abundant oil and gas resources, the significant position of shipping lane. The project of Nansha islands digital science education software promotes the dissemination of marine culture and awareness of the oceans and provides better marine cultural services to the public. Using NIDSES we can know the history, geography and the humanities the Nansha islands and it will be of great importance. The experimental results show that our platform processes rich multimedia resources, convenient communication cooperation, friendly interaction and other unique advantages, which provides the hybrid-type teaching and learning with efficient support.

\section{ACKNOWLEDGMENT}

This work was supported by Foundation of Humanities and Social Sciences Research in Ministry of Education, China (Construction and application of marine science popularization service platform of Nansha Islands in China. 
No. 16YJCZH038), and Characteristic innovation projects of universities in Guangdong (No. 2016GXJK101), and science research of Lingnan Normal University (No. LZL1509).

\section{REFERENCES}

[1] Nina Aschenbrenner, Leif Geiger. Transforming Scene Graphs Using Triple Graph Grammars-A Practice Report [J]. Lecture Notes in Computer Science, 2008, 5088:32-43.

[2] JoséMiguel Salles Dias,Pedro Nande, Nuno Barata,AndréCorreia. OGRE -Open Gestures Recognition Engine, a Platform for GestureBased Communication and Interaction [J]. Lecture Notes in Computer Science, 2006, 3881:129-132.

[3] Zhang H,Shi Y,Yuen DA,Yan Z,Yuan X,Zhang C. Modeling and visualization of tsunamis [J]. Pure and Applied Geophysics,2008,165:475-496.

[4] Poulter B,Halpin PN. Raster modeling of coastal flooding from sealevel rise [J]. International Journal of Geographical Information Science, 2008, 22:167-182

[5] Marc Herrlich,Ronald Meyer,Rainer Malaka and Helmut Heck. Development of a Virtual Electric Wheelchair Simulation and Assessment of Physical Fidelity Using the Unreal Engine [J]. Lecture Notes in Computer Science, 2010, 6243: 286-293.

[6] Daniel E, Atkins John B, John S B, et al.National educational technology plan,Office of Educational Technology U.S.Department of Education,2010, pp.101-103. 